\section{The management of primary rhegmatogenous retinal detachment not involving the fovea}

SF Ho ${ }^{1,2}$, A Fitt ${ }^{1}$, K Frimpong-Ansah ${ }^{1}$ and MT Benson ${ }^{1,2}$

\begin{abstract}
Aim To establish the likelihood of, and risk factors associated with, progression of 'macula-on' retinal detachment.

Methods A multi-centre prospective observational study of patients with acute retinal detachment, not involving the fovea, was conducted over a 6-month period. Data collected included duration of symptoms, visual acuity, presence of posterior vitreous detachment, retinal drawings and subretinal fluid (SRF) distance from the fovea at a minimum of two time points.
\end{abstract}

Results A total of 82 data sets from 15 institutions were analysed. Of 82 cases 11 (13\%) demonstrated progression of fluid. Mean progression in those cases which progressed was 2.3 disc diameters (dd) and the average rate of progression was $1.80 \mathrm{dd} / \mathrm{day}$. Binary regression analysis failed to reveal any statistically significant risk factors for progression. Multiple regression analyses were made to identify risk factors. With distance of SRF from fovea at operation as a function, distance of SRF at presentation was the only statistically significant risk factor. In all, $26 \%$ of patients underwent surgery out-ofhours. A total of $83 \%$ patients achieved a 6week best-corrected vision of $6 / 9$ or better. Conclusion Most retinal detachments in this study did not progress within the first few days. The distance of SRF from the fovea at presentation was the only statistically significant risk factor for progression to foveal detachment. Eye (2006) 20, 1049-1053. doi:10.1038/sj.eye.6702083; published online 21 October 2005

Keywords: 'macula-on' retinal detachment; progression; risk factor; duration of retinal detachment

\section{Introduction}

Traditionally, 'macula-on' retinal detachments (MORD) (ie those where the fovea is not involved at presentation) are managed on an urgent basis. This is largely based upon clinical experience; there is also evidence in medical literature, which suggests that a delay in surgery may result in the extension of the detachment to involve the macula, and hence a worse visual outcome for the patient. ${ }^{1,2}$ This has resource and logistical implications, since emergency surgery is in general more expensive $^{3}$ and it may require rescheduling of previously booked cases.

Although it is a standard clinical practice to differentiate between macula-on and macula-off detachment for the purpose of management, the medical literature is lacking in studies of the progression of macula-on to macula-off retinal detachment. Given the logistical and clinical implications of emergency surgery, it seems pertinent to examine the course of MORD and to look for factors indicating a high risk of rapid progression of the detachment. Thus, a prospective multi-centre observational study of progression of MORD was established. The aim of this pilot study was to identify risk factors for rapid progression of detachment, by analysing the clinical characteristics of MORD and recording the progression of these detachments over the period between diagnosis and surgery.

\section{Materials and methods}

The study, termed the 'macula-on retinal detachment (MORD) study', was a multi-centre prospective observational study of patients with MORD, from January to June 2001. Patients were included in the study if they had acute onset MORD (with symptoms of less than 3 months duration).
${ }^{1}$ Birmingham and Midland Eye centre, Birmingham, UK

${ }^{2}$ Birmingham Heartlands and Solihull Hospital, Birmingham, UK

Correspondence: SF Ho, Ophthalmology

Department,

Royal Victoria Hospital, Grosvenor Road, Belfast BT12 6BA, UK

Tel: + 4402890633612 ; Fax: + 4402890240503.

E-mail: s_ho2@

yahoo.com

Received: 22 April 2005 Accepted in revised form: 29 July 2005

Published online:

21 October 2005

None of the authors has any competing financial interest in the subject matter of the presentation

The paper was accepted as a poster for the ARVO meeting in May 2005 
Patients were excluded if they had chronic retinal detachment (with symptoms for more than 3 months). Those who had previous retinal detachment surgery in the affected eye were also excluded as these patients could arguably exhibit atypical progression. Surgery was to be carried out by or with the involvement of surgeons with a subspecialty interest in retinal surgery.

Other exclusion criteria included vision worse than $6 / 9$ in the affected eye at presentation (unless media opacity present), and history of reduced vision in the affected eye.

All consultant members of the British and Eire Association of Vitreoretinal Surgeons were invited to collaborate. Data were collected on a proforma, and included: age, gender, date of presentation, affected eye, duration and type of symptoms, visual acuity (VA) in both eyes, retinal drawing, subretinal fluid (SRF) distance from the centre of fovea in disc diameters, presence of posterior vitreous detachment (PVD), lens status, the presence of myopia, type of preoperative care including any use of posturing, day and date of operation, SRF distance from the fovea, and results of surgery at the 6-week follow-up. The SRF fluid distance was measured as the closest point of SRF to the fovea.

No attempt was made by the study organisers to influence the clinical management of cases. This was left to the discretion of the participating Ophthalmologists.

\section{Results}

A total of 15 ophthalmological units collaborated, with between 2 and 10 correct forms returned from each unit. In total 27 forms were rejected because of insufficient data, and 82 forms with adequate data were analysed. There were 50 male and 32 female patients included in the study. The mean patient age was 51 years (range 1853 years). In all, 40 of the affected eyes were right eyes and 42 were left eyes.

Regarding the retinal detachment configuration, 34 $(41.5 \%)$ of detachments were located superiorly/ superotemporally, 24 (29.3\%) inferiorly, eight (9.8\%) were temporal, six $(7.3 \%)$ superonasal, five $(6.1 \%)$ extensive ( $>2$ quadrant of retinal detachment) and five dual (two separate areas of SRF). In total, 45 patients (55\%) had at least one U-tear (including two giant retinal tears) and 36 patients $(45 \%)$ had non-U-tear retinal detachments (seven dialyses, 30 with round holes). PVD was present in $73.2 \%$ of patients. In all, $88 \%$ (72) of patients were phakic, $11 \%$ (9) were pseudophakic and one of the patients was aphakic.

A total of 50 patients $(61 \%)$ underwent surgery within 1 day of presentation; 10 patients (12\%) had surgery on day $2 ; 13$ patients $(16 \%)$ were operated upon between days 3 and 5; and 9 patients (11\%) underwent surgery from day 6 onwards. Of 82 patients, 54 (66\%) were asked to posture preoperatively. The average interval from presentation to surgery was 2.33 days (range 0-20 days).

Progression of the retinal detachment was gauged by assessing the SRF shift towards the fovea, comparing the distance between the closest SRF from the fovea at presentation and at operation. The overall average progression is $0.29 \mathrm{dd}$ with standard deviation (SD) of 1.08. Of the 82 cases analysed, only 11 cases demonstrated progression. Thus the probability of progression in this observational study was $11 / 82$ (0.1341) with a $95 \%$ confidence interval of $0.060-0.208$. With the 11 cases that progressed, the mean progression was $2.30 \mathrm{dd}$ with a SD of $1.90 \mathrm{dd}$ (range: $0.5-6 \mathrm{dd}$ ). Taking into account the interval between presentation and operation, the mean rate of progression was $1.80 \mathrm{dd} /$ day (SD: $1.58 \mathrm{dd} /$ day, range: $0.125-4.5 \mathrm{dd} /$ day). Logistic regression analysis (in which the end points were: retinal detachment which progressed $v$ s retinal detachment which did not progress) failed to reveal any statistically significant risk factors for progression. The factors analysed included the interval between presentation and operation, the interval between presence of symptoms and operation, the presence of PVD, quadrantic location of SRF, location of SRF above midline, presence of U-tear, presence of $U$-tear and at least one symptom of duration more than 7 days, posturing, myopia, gender and age.

Multiple regression analysis was used to analyse various factors possibly associated with the progression of SRF. The proximity of SRF to the fovea at the time of surgery was used as a function, and its relationship with other factors was analysed, including: the distance of SRF from the fovea at presentation; the quadrantic location of SRF; location of SRF above the midline; the presence of PVD; the interval between presentation and operation; the interval between presence of symptoms and operation; the presence of U-tears; the presence of U-tear and at least one symptom of duration more than 7 days; posturing; myopia; age and gender. The only statistically significant parameter found to be related with the proximity of SRF to the fovea at surgery was the distance of SRF from fovea at presentation (Table 1 and Figure 1).

SRF progressed towards the fovea in 11 patients $(13 \%)$. The average age of these particular patients was 58 years. The average rate of fluid shift was $1.80 \mathrm{dd} /$ day. Eight of these 11 patients $(73 \%)$ had superior retinal detachment, two patients $(18 \%)$ had temporal detachment, and one (9\%) had inferior detachment. Seven of these patients $(64 \%)$ had U-tears, and all of these tears were located between 10 and 2 o'clock positions. Four patients (36\%) had round holes only, and all of these had at least one hole above the horizontal midline. 9 of the 11 patients had PVD. On average, there were 1.91 retinal breaks. 


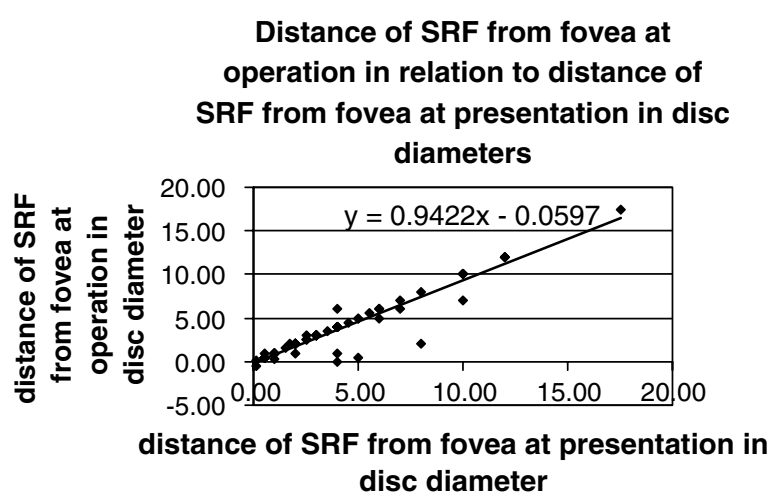

Figure 1 Distance of subretinal fluid from fovea at operation in relation to distance of subretinal fluid from fovea at presentation in disc diameters.

Table 1 Multiple regression analysis with proximity of subretinal fluid at operation in disc diameter as a function

\begin{tabular}{lc}
\hline Predictor & $\mathrm{P}$ \\
\hline $\begin{array}{l}\text { SRF distance from fovea at presentation } \\
\quad \text { (in disc diameter) }\end{array}$ & 0.000 \\
$\begin{array}{l}\text { Duration of retinal detachment (based on } \\
\quad \text { duration of symptoms and interval from }\end{array}$ & 0.714 \\
$\quad$ presentation to operation) (in days) & \\
Interval from presentation to operation (in days) & 0.604 \\
Superotemporal quadrant & 0.438 \\
Superonasal quadrant & 0.690 \\
Inferotemporal quadrant & 0.569 \\
Inferonasal quadrant & 0.600 \\
Posterior vitreous detachment & 0.786 \\
Location of subretinal fluid above midline & 0.343 \\
Presence of U-tear & 0.702 \\
U-tear and at least one symptom of more than 7 days & 0.726 \\
Posturing & 0.153 \\
Myopia & 0.726 \\
Gender & 0.211 \\
Age (years) & 0.895 \\
\hline
\end{tabular}

$S=0.69, R^{2}=96.1 \%$, Adjusted $R^{2}=96 \%$.

Nine of 11 patients $(82 \%)$ had been required to posture preoperatively. None of the units participating performed bilateral patching.

There were 71 patients in whom fluid did not progress towards the fovea, ( $87 \%$ of the study population). The average age of these patients was 50 years. The interval between presentation and surgery was 2.42 days on average (range: $0-20$ days). Of these 71 patients, 37 (52\%) had U-tears and 49(69\%) had PVD. On average, they had 2.32 retinal breaks.

The surgical technique employed was as follows: 53 patients $(64.6 \%)$ had conventional scleral buckling surgery; 27 (33\%) underwent pars plana vitrectomy; and two patients $(2.4 \%)$ had pneumoretinopexy. The success rate after one procedure was $92.7 \%$, with reoperation being carried out in six cases. During the 6-weeks
Table 2 Characteristics of three patients in whom macula was detached by the time of operation

\begin{tabular}{|c|c|c|c|}
\hline & Patient 1 & Patient 2 & Patient 3 \\
\hline Age (years) & 59 & 72 & 76 \\
\hline Duration of symptom & 1 day & 2 days & 1 day \\
\hline $\begin{array}{l}\text { Location of subretinal } \\
\text { fluid (in clock } \\
\text { hour positions) }\end{array}$ & $11.30-1.45$ & $2.30-8.00$ & $1.00-3.00$ \\
\hline U-tear/hole & $\begin{array}{l}1 \text { U-tear } \\
\text { (12.15) }\end{array}$ & $\begin{array}{l}4 \text { holes } \\
(2.15,6.00 \\
6.00,9.45)\end{array}$ & $\begin{array}{l}1 \text { U-tear } \\
(1.45)\end{array}$ \\
\hline $\begin{array}{l}\text { Distance of subretinal } \\
\text { fluid from fovea } \\
\text { at presentation } \\
\text { (in disc diameter) }\end{array}$ & 4 & 0.5 & 0.1 \\
\hline $\begin{array}{l}\text { Days after presentation } \\
\text { in which macular } \\
\text { detachment was found }\end{array}$ & $\begin{array}{l}1 \text { (macula } \\
\text { split) }\end{array}$ & 4 & 1 \\
\hline Posturing performed & Yes & Yes & Yes \\
\hline $\begin{array}{l}\text { 6/52 postoperative } \\
\text { best-corrected } \\
\text { visual acuity }\end{array}$ & $6 / 18$ & $6 / 9$ & $6 / 12$ \\
\hline
\end{tabular}

postoperative data collection period, one patient had persistent detachment and one had a 'puddle' of SRF. The rate of reattachment was $97.5 \%$ at 6 weeks post surgery.

Of 82 patients, $21(26 \%)$ underwent surgery outside normal working hours (out-of-hours implies anaesthetic or procedure commencing between 1801 and 0759 hours, or on a Saturday, Sunday or bank holiday). Nine patients had operations on Saturday, three on Sunday, and nine on weekday evenings.

Of 82 patients, $68(83 \%)$ achieved best-corrected VA of $6 / 9$ or better at 6 weeks. Seven patients $(8.5 \%)$ achieved 6/12, two (2.4\%) achieved 6/18, two (2.4\%) achieved $6 / 24$, and three $(3.6 \%)$ achieved VA of $6 / 36$. The reasons for reduction in central VA (VA of $6 / 18$ or less) were as follows: $4 / 7$ (57\%) had nuclear sclerosis, $1 / 7$ (14.3\%) had posterior capsular opacity, $1 / 7$ (14.3\%) had cystoid macular oedema and 1/7 (14.3\%) had possible silicone oil toxicity.

Three patients were found to have macular detachment at operation. In one case, the macula detached on the day of presentation, and in another the macula was bisected by SRF within 1 day of presentation. The third case suffered macular detachment by day 4 having presented with SRF located $0.5 \mathrm{dd}$ from the fovea. Final VA in these patients ranged from $6 / 9$ to $6 / 18$ (Table 2).

\section{Discussion}

MORD is traditionally viewed as a surgical emergency. It is a widely held view that such cases should be operated 
upon as soon as possible; preferably within 24 hours. ${ }^{4}$ This approach has a clear impact on resources and organisation of service. Emergency surgery is more costly than planned surgery, particularly when surgeons' preferences are considered. ${ }^{3}$ It affects surgical planning, with the cancellation of preexisting cases. In all, $26 \%$ of the operations performed in this study took place outside normal working hours. Patients with coexisting medical problems may be exposed to greater anaesthetic risk, particularly if they are inadequately assessed in an emergency setting.

The issue of MORD has become a more pressing concern with the recent move towards subspecialisation and tertiary referral in the United Kingdom. A recent study in Liverpool suggested that there has been an increase in the number of cases performed in specialist vitreoretinal units. ${ }^{5}$

This study highlights the relatively 'benign' nature of the majority of MORD and it raises the issue of whether same-day surgery is necessary for most patients. Only $13 \%$ of the reported patients had documented progression of SRF towards the fovea over the study period. For those who did demonstrate progression, this was at a mean rate of $1.8 \mathrm{dd} /$ day, which is a noticeable (and therefore easily documented) change. Tani et $a l^{6}$ have noted that some eyes maintain macular attachment in spite of a relatively long duration of peripheral detachment, and postulate a more benign subgroup of detachments, which are less likely to have dynamic proliferative factors leading to extension of detachment, macular pucker, or ultimate failure. Daily observation of SRF distribution may be an option for patients who are not undergoing same-day surgery.

The analysis of risk factors associated with progression of SRF indicated that the distance of SRF from fovea at operation was associated with the distance of SRF from the fovea at presentation, which was foreseen. Other factors such as the presence of PVD, quadrantic location of SRF, and importantly the interval between presentation and operation were not found to be statistically significant. This suggests that in acute MORD the decision to carry out urgent surgery should be primarily based upon the proximity of SRF to the fovea. Analysis of the three patients with macular detachment at operation supports this hypothesis. Two patients with macular detachment had SRF located between 0.1 and $0.5 \mathrm{dd}$ from the fovea at presentation.

It is generally expected that postoperative VA should be the same as the preoperative VA for MORD. The reduction of VA to 6/18 or worse that was observed in seven patients was related to the surgery carried out in all cases, and no reduction in vision to this level was the result of macular detachment.
Preoperative posturing was frequently carried out in the study patients. The study was not designed to determine the efficacy of this measure, but it is the clinical impression of the authors that it does help to discourage SRF progression in some patients.

\section{Conclusion}

We accept that there are several limitations to this pilot study. This was an observational study, and not randomised, although it was prospective. There was significant potential for interobserver variation particularly with regard to SRF distance from the fovea. The study was open to bias on the part of observers, and to selection bias. Furthermore, the sample size was small, and this may have limited the identification of risk factors for progression to macula-off detachment. The study was set up as an informal pilot study, and may well not have included all patients in the participating departments (eg there were larger units, where not all subspecialists were participating). If this study was used to stimulate a more formal study on a wider basis then this defect could be addressed.

We believe, however, that this study indicates that most MORD do not show rapid progression and thus do not necessarily require immediate surgical treatment. Close proximity of SRF to the fovea at presentation is an indicator of high risk of progression to macular detachment, particularly when the fluid is within $1 \mathrm{dd}$ of the fovea. In other cases, we argue that it would be reasonable to observe for SRF progression until surgery can be carried out in a scheduled operating session during normal working hours. Preoperative posturing may or may not have its place in the management of these patients.

We suggest that this pilot study supports the establishment of a more rigorous formal study involving more Ophthalmic institutions.

\section{Acknowledgements}

We thank Mr Andrew Callear, Mr Bartley McNeela, Mr Chrisjan Dees, Mr Charles James, Mrs Diana Depla, Mr David Inglesby, Mr David Steel, Mr David Cottrell, Mr Richard Bates, Mr James West, Miss Karen Goodall, Mr Peter Simcock, Mr Richard Goble, Mr Roger Gray, Mrs Brenda Billington, Mr Patrick Richardson, Mr Kevin Stannard, Mr. Steve Charles and Mr Tony Casswell for their help with the study. We also thank Mr Tim Marshall in Department of Public Health in University of Birmingham who assisted us in the statistical analysis of this paper. 


\section{References}

1 Burton TC. Recovery of visual acuity after retinal detachment involving the macula. Trans Am Ophthalmol Soc 1982; 80: $475-477$.

2 Ross WH, Kozy DW. Visual outcome in macula-off rhegmatogenous retinal detachments. Ophthalmology 1998; 105: 2149-2153.

3 Hartz AJ, Burton TC, Gottlieb MS, McCarty DJ, Williams DF, Prescott A et al. Outcome and cost analysis of scheduled vs emergency scleral buckling surgery. Ophthalmology 1992; 99: 1358-1363.
4 American Academy of Ophthalmology. Ophthalmic procedure assessment. The repair of rhegmatogenous retinal detachment. Ophthalmology 1995; 103: 1313-1324.

5 Ah-Fat FG, Sharma MC, Majid MA, McGalliard JN, Wong $\mathrm{D}$. Trends in vitreoretinal surgery at a tertiary referral centre: 1987-1996. Br J Ophthalmol 1999; 83: 396-398.

6 Tani P, Robertsib DM, Langworthy A. Rhegmatogenous retinal detachment without macular involvement treated with scleral buckling. Am J Ophthalmol 1980; 90: 503-508. 